Research article

\title{
Phosphodiesterase-5 inhibitors have distinct effects on the hemodynamics of the liver \\ Leonie Halverscheid ${ }^{1}$, Peter Deibert ${ }^{2}$, René Schmidt ${ }^{3}$, Hubert E Blum ${ }^{4}$, Torsten Dunkern ${ }^{5}$, Benedikt HJ Pannen ${ }^{1}$ and Wolfgang Kreisel*4
}

\begin{abstract}
Address: ${ }^{1}$ Department of Anesthesiology, University Hospital Duesseldorf, Germany, ${ }^{2}$ Department of Preventive and Rehabilitative Sport Medicine, University Hospital Freiburg, Germany, ${ }^{3}$ Department of Anesthesiology and Critical Care Medicine, University Hospital Freiburg, Germany, ${ }^{4}$ Department of Medicine II, University Hospital Freiburg, Germany and ${ }^{5}$ Department of Biochemistry, Nycomed GmbH, Konstanz, Germany

Email: Leonie Halverscheid - halverscheidl@anesthesia.ucsf.edu; Peter Deibert - peter.deibert@uniklinik-freiburg.de; René Schmidt - rene.schmidt@uniklinik-freiburg.de; Hubert E Blum - hubert.blum@uniklinik-freiburg.de;

Torsten Dunkern - torsten.dunkern@nycomed.com; Benedikt HJ Pannen - benedikt.pannen@med.uni-duesseldorf.de;

Wolfgang Kreisel* - wolfgang.kreisel@uniklinik-freiburg.de

* Corresponding author
\end{abstract}

Published: 18 September 2009

BMC Gastroenterology 2009, 9:69 doi:10.1 186/147|-230X-9-69

This article is available from: http://www.biomedcentral.com/I47I-230X/9/69

2009 Halverscheid et al; licensee BioMed Central Ltd.

This is an Open Access article distributed under the terms of the Creative Commons Attribution License (http://creativecommons.org/licenses/by/2.0), which permits unrestricted use, distribution, and reproduction in any medium, provided the original work is properly cited.
Received: 6 April 2009

Accepted: 18 September 2009

\begin{abstract}
Background: The NO - cGMP system plays a key role in the regulation of sinusoidal tonus and liver blood flow with phosphodiesterase-5 (PDE-5) terminating the dilatory action of cGMP. We, therefore, investigated the effects of PDE-5 inhibitors on hepatic and systemic hemodynamics in rats.

Methods: Hemodynamic parameters were monitored for $60 \mathrm{~min}$. after intravenous injection of sildenafil and vardenafil [I, I0 and I00 $\mathrm{g} / \mathrm{kg}$ (sil I, sil I0, sil I00, var I, var I0, var I00)] in anesthetized rats.

Results: Cardiac output and heart rate remained constant. After a short dip, mean arterial blood pressure again increased. Systemic vascular resistance transiently decreased slightly. Changes in hepatic hemodynamic parameters started after few minutes and continued for at least 60 min. Portal (varlo -31\%, sill $0-34 \%$ ) and hepatic arterial resistance (varlo -30\%, sill $0-32 \%$ ) decreased significantly $(p<0.05)$. At the same time portal venous (varl $0+29 \%$, sill $0+24 \%)$, hepatic arterial (var $10+34 \%$, sill $0+48 \%$ ), and hepatic parenchymal blood flow (var $10+15 \%$, sill $0+15 \%)$ increased significantly $(p<0.05)$. The fractional liver blood flow (total liver flow/cardiac output) increased significantly (varlo 26\%, sill $023 \%$ ). Portal pressure remained constant or tended to decrease. 10 $\mu \mathrm{g} / \mathrm{kg}$ was the most effective dose for both PDE-5 inhibitors.
\end{abstract}

Conclusion: Low doses of phosphodiesterase-5 inhibitors have distinct effects on hepatic hemodynamic parameters. Their therapeutic use in portal hypertension should therefore be evaluated. 


\section{Background}

Nitric oxide (NO) plays a crucial role in hepatic microvascular blood flow under physiological conditions [1-5]. Hepatic vascular resistance is regulated on the one hand by contraction or relaxation of smooth muscle cells in the terminal arterioles. On the other hand, perisinusoidal stellate cells (Ito-cells) regulate sinusoidal tonus depending on concentration of NO synthesized by the sinusoidal endothelial cells. The diameter of liver sinusoids is responsible for up to $1 / 3$ of the intrahepatic vascular resistance and is regulated by an interplay of endothelial cells, hepatocytes and stellate cells $[6,7]$. NO is synthesized by endothelial cells and activates soluble guanylate cyclase of stellate cells. This results in the formation of cGMP that regulates the tonus of stellate cells and sinusoids $[8,9]$. This action is terminated by phosphodiesterase-5 (PDE-5), which converts cGMP to 5'-GMP [10,11]. Furthermore, vascular tonus depends on the differential distribution of $\alpha$ - and $\beta$-receptors in the blood vessels. Angiotensin II and humoral factors, e.g., endothelins, with strong vasoconstrictor effects within extrasinusoidal and sinusoidal sites contribute to the regulation of liver blood flow [12-14]. Recently it was shown that induction of heme oxygenase-1 may reduce ischemia/reperfusion injury, probably by enhancing microvascular blood flow [15]. Data from the same group suggest an interplay between hepatic NO synthesis and heme oxygenase-1 regulation [16].

In liver cirrhosis, the NO - cGMP system is dysregulated. Portal hypertension is caused by an increased intrahepatic vascular resistance resulting from the disturbed liver architecture, perisinusoidal fibrosis, and cellular alterations of liver sinusoids as well as from functional changes. Due to a reduced activity of the endothelial NO synthase (eNOS) in liver endothelial cells NO decreases whereas hepatic stellate cells transform to contractile myofibroblasts $[5,7,17-21]$. These factors and an increased PDE-5 activity in liver cirrhosis result in the contraction of sinusoids [2225]. In contrast to the intrahepatic condition in the splanchnic vascular system, NO production increases causing dilation of the mesenteric blood vessels and splanchnic hyperperfusion $[5,26,27]$. Apart from liver cirrhosis, an altered NO metabolism also occurs in other clinical settings, such as ischemia and reperfusion injury during liver surgery $[1,28,29]$. Several animal studies have shown that a selective modulation of NO metabolism in the liver reduces intrahepatic resistance and portal pressure in cirrhosis [30-36].

It is intriguing to investigate whether PDE-5 inhibitors which inhibit the conversion of cGMP to 5'-GMP could dilate hepatic sinusoids and increase hepatic blood flow. In a previous clinical pilot study we showed that the PDE5 inhibitor vardenafil increases portal venous flow in nor- mal and cirrhotic liver and lowers portal pressure and hepatovenous pressure gradient in cirrhotics [37]. In a patient with portopulmonary hypertension we could further demonstrate that the PDE-5 inhibitor tadalafil lowers both pulmonary arterial and portal pressure [38]. Recently, Lee et al. showed that after a standard dose of 50 mg sildenafil hepatic production of cyclic guanosine monophosphate increases leading to a significant decrease of hepatic sinusoid resistance (34). These authors found no change in HVPG [39]. Clemmesen et al. [40] observed a $>10 \%$ decrease of HVPG in 4 of 10 patients with liver cirrhosis. However, from animal experiments [41] and case reports [42-44] it was considered that PDE-5 inhibitors may even increase portal pressure.

The conflicting results obtained with PDE 5 inhibitors in the clinical setting require a thorough investigation in an experimental model prior to proceed to large scale clinical studies. Neither the optimal dose of PDE 5 inhibitors nor the optimal parameters of efficacy are known for a potential use of these drugs in liver cirrhosis. In this study we analyzed the effects of PDE-5 inhibitors on hemodynamics of normal liver in rats. We collected exact measurements of the effects of sildenafil and vardenafil, respectively, on hepatic blood flow and vascular resistances, portal venous pressure, and regional hepatic perfusion as well as systemic hemodynamic variables, e.g. cardiac output.

\section{Methods \\ Reagents}

Isoflurane was purchased from Abbott (Wiesbaden, Germany), pancuronium from Organon (BH Oss, Netherlands). Sildenafil and vardenafil were obtained from the Nycomed GmbH, (Konstanz, Germany). They were dissolved in $0.9 \% \mathrm{NaCl}$, containing $0.04 \% 0.1 \mathrm{~N} \mathrm{HCl}$.

\section{Animals}

Overnight fasted male Sprague Dawley rats (Charles River, Sulzfeld, Germany) weighing $388 \pm 37 \mathrm{~g}$ were used for all experiments. The experimental protocol was approved by the local Animal Care and Use Committee. All animals received care according to the Guide for the Care and Use of Laboratory Animals (American Association for Laboratory Animal Science. MD: NIH 1985).

\section{Animal preparation}

After inhalational induction of anesthesia with isoflurane a tail vein was cannulated and a tracheotomy was performed. After muscle relaxation by intravenous injection of pancuronium $(0.1 \mathrm{mg} / \mathrm{kg}$ i.v. $)$ the animals were mechanically ventilated (Rodent Ventilator UB 7025-10, Harvard Apparatus, March-Hugstetten, Germany), under continued isoflurane anesthesia. For compensation of evaporative losses during the initial procedure of surgical 
preparation $4 \mathrm{ml} / \mathrm{kg} / \mathrm{h}$ of a crystalloid solution (Jonosteril ${ }^{\circledast}$, Fresenius, Bad Homburg, Germany) were continuously infused. An arterial line (polyethylene PE-50 tube) was placed into the left femoral artery for blood pressure monitoring and blood withdrawal. For cardiac output analysis by the transpulmonary thermodilution technique, a thermistor tip catheter $(9490 \mathrm{E}$, Columbus Instruments, Columbus, OH, USA) was inserted into the aortic arch through the left carotid artery. For monitoring central venous pressure and injection of saline at $4^{\circ} \mathrm{C}$ to measure cardiac output, a PE-50 catheter was positioned close to the right atrium via the right external jugular vein. At the time of laparotomy the continuous infusion of Jonosteril was increased from 4 to $10 \mathrm{ml} / \mathrm{kg} / \mathrm{h}$. Ultrasound flow probes were placed at the common hepatic artery and the portal vein (T206, small animal flow meter, Transonic, Ithaca, NY, USA). In addition, the portal vein was cannulated (26G, Insyte-W, BD, USA) to measure portal pressure. For microvascular (parenchymal) blood flow measurement, a microvascular flow probe (DP10M 100ST for DRT-4 Laser Doppler Monitor, Moor Instruments Ltd., Axminster, UK) was placed in a defined position on the surface of the left liver lobe. The body temperature was maintained normothermic $\left(37 \pm 0.5^{\circ} \mathrm{C}\right)$ throughout the experiment.

\section{Experimental Protocol}

After stabilization for $15 \mathrm{~min}$ after surgery, baseline hemodynamic parameters were measured. Animals were then randomized into the following groups: sil100 100 $\mu \mathrm{g} / \mathrm{kg}(\mathrm{n}=7)$, sil10 $10 \mu \mathrm{g} / \mathrm{kg}(\mathrm{n}=7)$, sil1 $1 \mu \mathrm{g} / \mathrm{kg}(\mathrm{n}=5)$, va100r $100 \mu \mathrm{g} / \mathrm{kg}(\mathrm{n}=6), \operatorname{var} 1010 \mu \mathrm{g} / \mathrm{kg}(\mathrm{n}=7), \operatorname{var} 11$ $\mu \mathrm{g} / \mathrm{kg}(\mathrm{n}=5)$, controls $1 \mathrm{ml} / \mathrm{kg} 0.9 \% \mathrm{NaCl}$ containing $0.04 \% 0.1 \mathrm{~N} \mathrm{HCl}(\mathrm{n}=7)$. In order to minimize plasma volume related alterations of hemodynamic parameters the pharmacological intervention was carried out in a standard volume of $100 \mu \mathrm{l} / \mathrm{kg}$ injected over 90 seconds via the tail vein. Heart rate, mean arterial blood pressure, central venous pressure, portal venous pressure, portal venous flow, hepatic arterial flow, hepatic parenchymal flow and cardiac output were measured 0.5, 1, 3, 5, 10, 20, 30,45 and $60 \mathrm{~min}$ after injection. From the data we calculated the "fractional liver flow": Arterial liver flow + portal liver flow/cardiac output. It represents the proportion of cardiac output passing through the liver.

\section{Data analysis}

Normal distribution of all variables was tested before statistical analyses using the Kolmogorov-Smirnov test procedure. Changes between baseline and $60 \mathrm{~min}$ were analyzed using Wilcoxon rank sum test. Statistical differences between the groups at baseline were determined using a Kruskal-Wallis test on ranks. All p-values were two-sided and a p-value of $<0.05$ was considered statisti- cally significant. Analyses were performed using the SPSS software (version 15.0).

\section{Results}

The effects of sildenafil and vardenafil on hemodynamic parameters are presented in Table 1, Figure 1, Figure 2, and Figure 3. Table 1 shows data at baseline and $60 \mathrm{~min}$ after injection of sildenafil or vardenafil at the three concentrations, compared to controls. The 60 min time point was chosen because preliminary data had demonstrated that after $60 \mathrm{~min}$ a steady state is reached. Figure 1 depicts the time-dependent course of the two most important hepatic hemodynamic parameters, portal flow and portal pressure, at the three different doses of sildenafil and vardenafil. These curves show 1 . that the $10 \mu \mathrm{g} / \mathrm{kg}$ doses seem to induce the most impressive increase of portal blood flow, and 2. that despite increasing portal flow the portal pressure does not increase. Therefore, we depict the courses of further systemic and hepatic hemodynamic variables at this dose in Figure 2 and 3.

\section{Effect of sildenafil and vardenafil on systemic hemodynamic parameters}

In the control group, no parameter showed a significant change as compared to baseline (Table 1). Sil and var at 1 $\mu \mathrm{g} / \mathrm{kg}$ and $10 \mu \mathrm{g} / \mathrm{kg}$ induced a slight but significant change of mean arterial pressure (MAP) by $1.1--11.0 \%$. Systemic vascular resistance (SVR) decreased significantly in the sil10 and all vardenafil groups by $7.7-11.6 \%$. Most importantly, in none of the groups heart rate or cardiac output changed significantly, there was a short initial increase of heart rate and cardiac output after injection in all of the groups. Taken together, sildenafil and vardenafil induced only minor changes in systemic hemodynamic parameters.

\section{Effect of sildenafil and vardenafil on hepatic hemodynamic parameters}

Hepatic arterial resistance decreased by $17.4-32.2 \%$ in all intervention groups except in sil1. Consistent with these findings hepatic arterial blood flow increased in all intervention groups, reaching statistical significance in the sil10 (increase by $47.5 \%$ ), var10 and var100 groups (increase by $33.5 \%$ and $20.2 \%$ ), respectively. Portal resistance (calculated as portal pressure minus central venous pressure divided by portal flow) significantly decreased in var1, var10, var100, and sil10 groups by $22.0-38.6 \%$. Portal venous flow increased significantly with sil and var at 10 and $100 \mu \mathrm{g} / \mathrm{kg}$, respectively. Sil and var at $10 \mathrm{ug} / \mathrm{kg}$ increased portal flow by $24.1 \%$ and $29.2 \%$, respectively. Hepatic parenchymal blood flow significantly increased in all sil groups (by $6.3-15.3 \%$ ) and in the var10 and var100 groups (by $15.0 \%$ and $28.8 \%$ ). Taken together, in animals with normal liver we observed a decrease of arterial hepatic and portal (transhepatic) resistance and an 

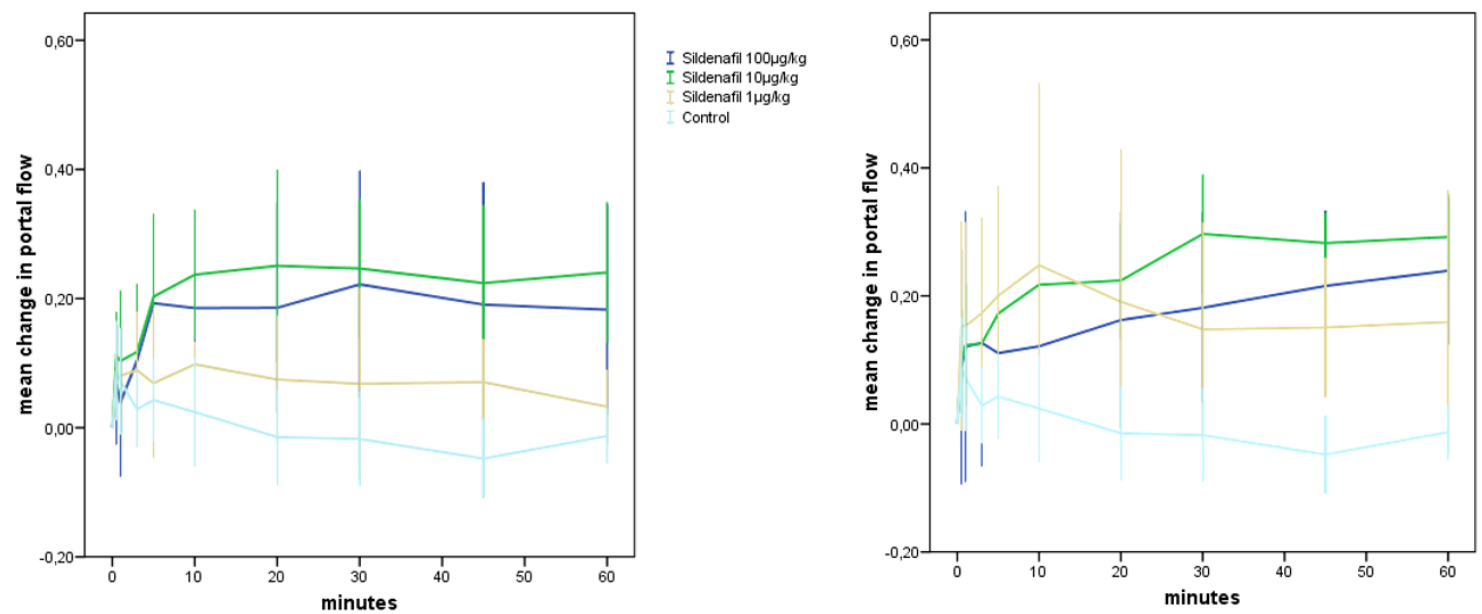

I Vardenafil $100 \mathrm{\mu g} \mathrm{kg}$ I Vardenafil $10 \mathrm{\mu g} / \mathrm{kg}$ Vardenafil $1 \mu \mathrm{g} / \mathrm{g}$
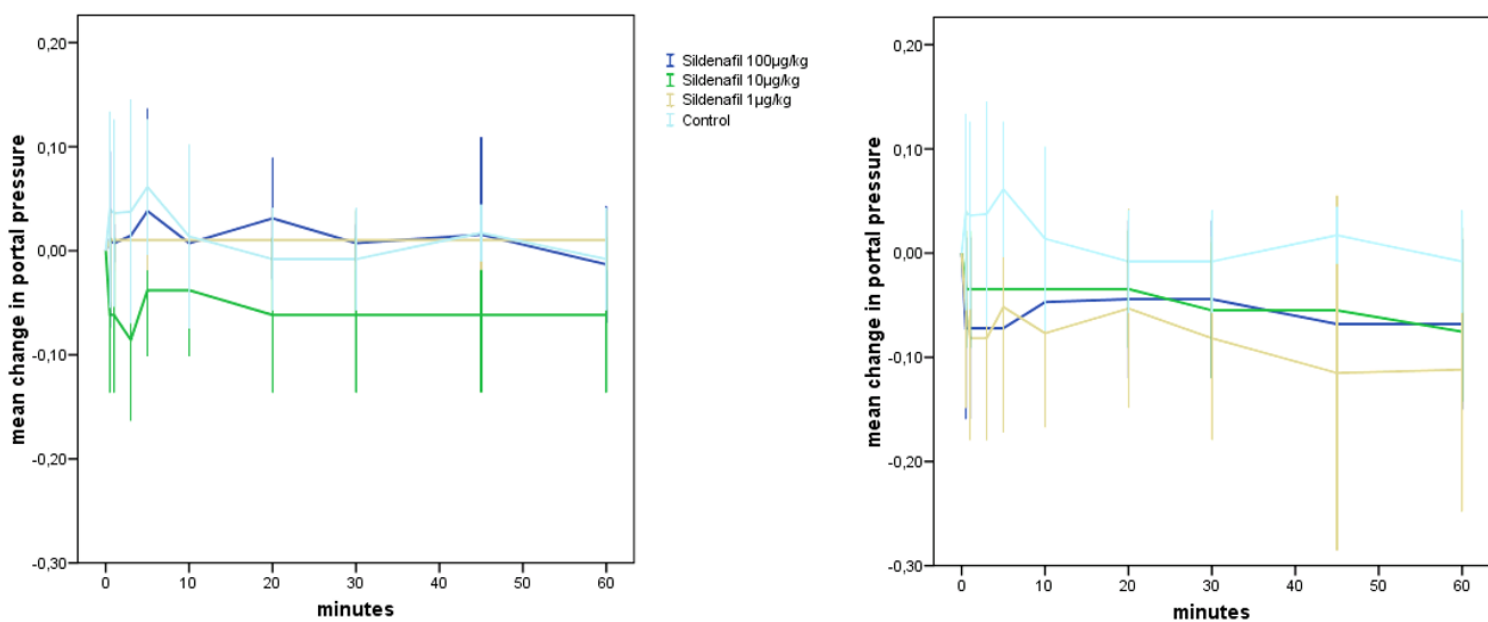

I Vardenafil $100 \mathrm{\mu g} / \mathrm{kg}$ I Vardenafil $10 \mu g \mathrm{~kg}$ Vardenafil $1 \mu \mathrm{g} / \mathrm{kg}$ Control

Figure I

Course of relative changes of portal flow and portal pressure after slow intravenous injection of different doses of sildenafil, vardenafil, or $0.9 \% \mathrm{NaCl}$. Ordinate: time (minutes) Abscissa: relative change (e.g. 0.00 means baseline; 0.10 means: increase by $10 \%$; -0.10 means: decrease by $10 \%)$. The values are indicated as mean $\pm 95 \%$ confidence intervals. Panel I: Portal flow. Panel 2: Portal pressure. The values are indicated as mean $\pm 95 \%$ confidence intervals. The curves at different doses are marked by colours.

increase of portal, arterial and parenchymal blood flow of the liver.

Most importantly, in none of the intervention groups portal pressure increased. To the contrary, in sil10, sil100, var1, var10, and var100 portal pressure decreased and nearly reached statistical significance in the var10 group $(\mathrm{p}=0.06)$.

Additional information can be derived from Figure 2 and Figure 3. Immediately after injection of sil or var at $10 \mu \mathrm{g} /$ kg MAP decreased (Figure 2, panel 1) but increased again only a few min later without reaching baseline levels at 60 $\mathrm{min}$. This pattern was observed in all intervention groups. SVR also dropped and then gradually increased without complete normalization at the end of the experiment (Figure 2, panel 2). PDE-5 inhibitors had no effect on the heart rate (data not shown) or cardiac output (Figure 2, panel 3).

Already a few minutes after the injection of sil or var hepatic arterial flow (Figure 3, panel 1), hepatic parenchymal flow (Figure 3, panel 2), and portal venous flow (Figure 1) increased. This increase was independent from cardiac output (which remained constant) and the slight drop of MAP. The hepatic effects became even more pronounced with time, e. g., after 15 min when drug distribution should have reached a steady state. Despite increased portal venous and hepatic arterial blood flow portal pressure does not increase. On the contrary, there is a trend to 


\section{Sildenafil $10 \mu \mathrm{g} / \mathrm{kg} \quad$ Vardenafil $10 \mu \mathrm{g} / \mathrm{kg}$}

Control
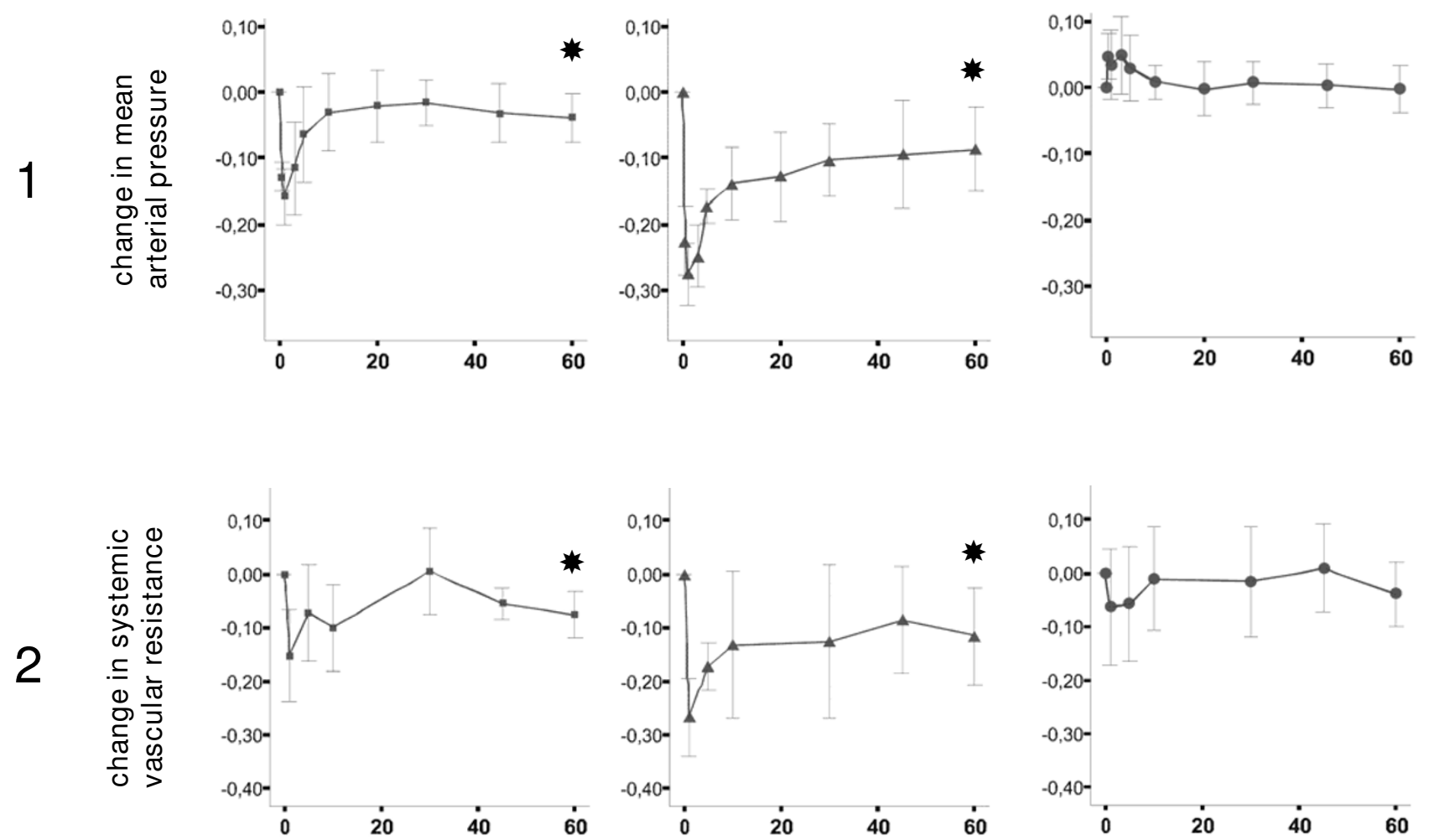

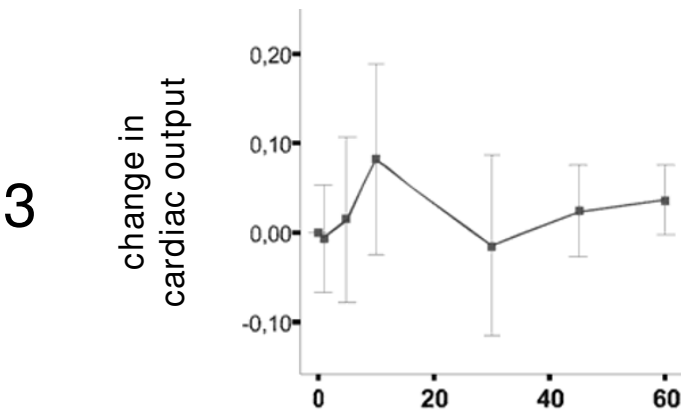

minutes

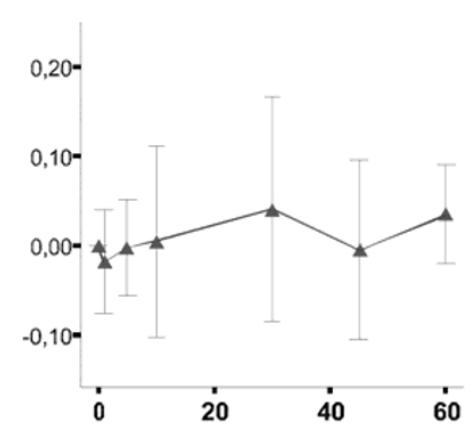

minutes

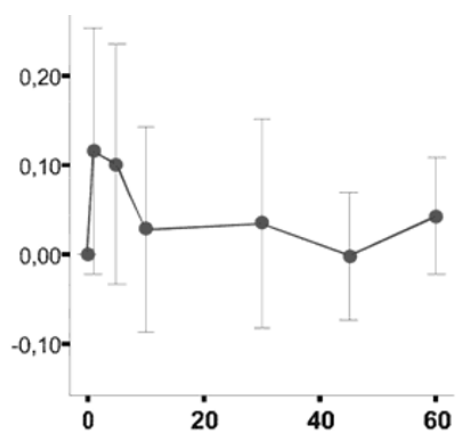

minutes

Figure 2

Course of relative changes of systemic hemodynamic parameters after slow intravenous injection of $10 \mu \mathrm{g} / \mathrm{kg}$ sildenafil or vardenafil or $\mathbf{0 . 9 \%} \mathbf{~ N a C l}$. Ordinate: time (minutes). Abscissa: relative change (e.g. 0.00 means baseline; 0.10 means: increase by $10 \%$; -0.10 means: decrease by $10 \%$ ). The values are indicated as mean $\pm 95 \%$ confidence intervals. Significant changes at $60 \mathrm{~min}(\mathrm{p}<0.05$; Wilcoxon rank sum test) are marked with black stars. Panel I: Mean arterial pressure. Panel 2: Systemic vascular resistance. Panel 3: Cardiac output. 

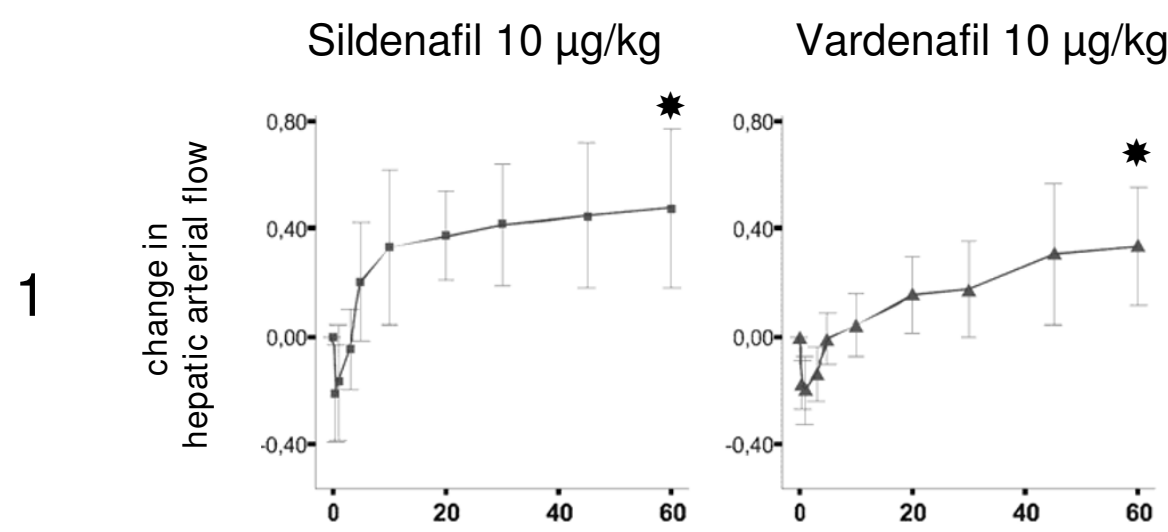

Control
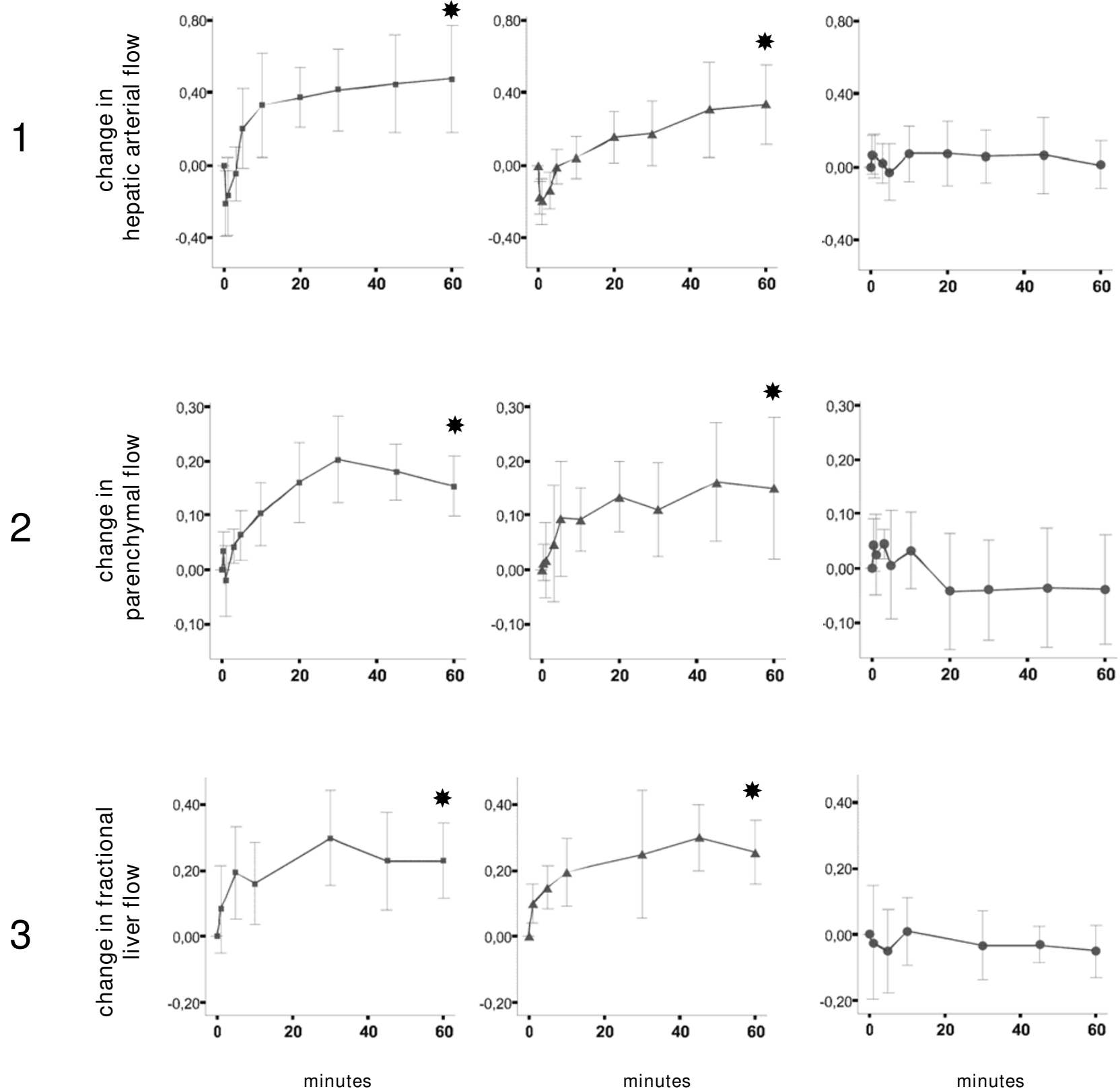

Figure 3

Course of relative changes of hepatic hemodynamic parameters after slow intravenous injection of $10 \mu g / k g$ sildenafil or vardenafil or $\mathbf{0 . 9 \% ~} \mathrm{NaCl}$. Ordinate: time (minutes). Abscissa: relative change (e.g. 0.00 means baseline; 0.10 means: increase by $10 \%$; -0.10 means: decrease by $10 \%$ ). The values are indicated as mean $\pm 95 \%$ confidence intervals. Significant changes at 60 min ( $p<0.05$; Wilcoxon rank sum test) are marked with black stars. Panel I: Hepatic arterial flow. Panel 2: Parenchymal flow. Panel 3: Fractional liver flow. 
Table I: Baseline parameters and relative changes after $60 \mathrm{~min}$, expressed as $\%$ of the baseline value

\begin{tabular}{|c|c|c|c|c|c|c|c|c|c|c|c|c|c|c|}
\hline $\begin{array}{l}\text { parameter } \\
\text { MAP }\end{array}$ & \multicolumn{2}{|c|}{ control } & \multicolumn{2}{|c|}{$\begin{array}{l}\text { sildenafil } \\
100 \mu \mathrm{g} / \mathrm{kg}\end{array}$} & \multicolumn{2}{|c|}{$\begin{array}{l}\text { sildenafil } \\
10 \mu \mathrm{g} / \mathrm{kg}\end{array}$} & \multicolumn{2}{|c|}{$\begin{array}{c}\text { sildenafil } \\
\mathrm{I} \mu \mathrm{g} / \mathbf{k g}\end{array}$} & \multicolumn{2}{|c|}{$\begin{array}{l}\text { vardenafil } \\
100 \mu \mathrm{g} / \mathrm{kg}\end{array}$} & \multicolumn{2}{|c|}{$\begin{array}{c}\text { vardenafil } \\
10 \mu \mathrm{g} / \mathrm{kg}\end{array}$} & \multicolumn{2}{|c|}{$\begin{array}{c}\text { vardenafil } \\
\text { I } \mu \mathrm{g} / \mathbf{k g}\end{array}$} \\
\hline $\begin{array}{l}\text { MAP } \\
(\mathrm{mmHg})\end{array}$ & $\begin{array}{l}84.19 \\
{[80.3-} \\
88.0]\end{array}$ & $\begin{array}{c}-0.3 \\
{[-3.8-} \\
3.2]\end{array}$ & $\begin{array}{c}85.0 \\
{[80.2-} \\
89.7]\end{array}$ & $\begin{array}{c}-2.6 \\
{[-6.5-} \\
1.2]\end{array}$ & $\begin{array}{c}84.9 \\
{[81.7-} \\
88.2]\end{array}$ & $\begin{array}{l}-3.9 * \\
{[-7.6-} \\
-0.0]\end{array}$ & $\begin{array}{c}83.9 \\
{[81.2-} \\
86.5]\end{array}$ & $\begin{array}{l}1.1 * \\
{[0.4-} \\
1.9]\end{array}$ & $\begin{array}{c}82.9 \\
{[79.7-} \\
86.2]\end{array}$ & $\begin{array}{c}-7.4 \\
{[-16.3-} \\
1.4]\end{array}$ & $\begin{array}{c}80.9 \\
{[77.5-} \\
84.3]\end{array}$ & $\begin{array}{l}-8.7 * \\
{[-15.0} \\
--2.4]\end{array}$ & $\begin{array}{c}88.0 \\
{[82.5-} \\
93.5]\end{array}$ & $\begin{array}{c}-11.0 \\
* \\
{[-16.7} \\
--5.3]\end{array}$ \\
\hline $\begin{array}{l}\text { HR } \\
(1 / \mathrm{min})\end{array}$ & $\begin{array}{r}366.4 \\
{[348.8-} \\
384.1]\end{array}$ & $\begin{array}{c}-1.1 \\
{[-2.9-} \\
0.7]\end{array}$ & $\begin{array}{l}375.0 \\
{[351.0-} \\
399.0]\end{array}$ & $\begin{array}{c}-1.9 \\
{[-3.0-} \\
6.7]\end{array}$ & $\begin{array}{c}375.7 \\
{[364.3-} \\
387.2]\end{array}$ & $\begin{array}{c}3.2 \\
{[-0.0-} \\
0.1]\end{array}$ & $\begin{array}{c}366.0 \\
{[332.2-} \\
399.8]\end{array}$ & $\begin{array}{c}2.9 \\
{[-2.1-} \\
3.9]\end{array}$ & $\begin{array}{c}368.3 \\
{[353.3-} \\
383.4]\end{array}$ & $\begin{array}{c}2.4 \\
{[-1.3-} \\
6.2]\end{array}$ & $\begin{array}{l}362.9 \\
{[353.3-} \\
372.4]\end{array}$ & $\begin{array}{c}5.2 \\
{[-3.4-} \\
13.7]\end{array}$ & $\begin{array}{c}346.0 \\
{[324.3-} \\
367.7]\end{array}$ & $\begin{array}{c}1.3 \\
{[-3.4-} \\
6.0]\end{array}$ \\
\hline $\begin{array}{l}\text { SVR } \\
(\mathrm{mmHg} / \\
\mathrm{ml} \cdot \mathrm{min})\end{array}$ & $\begin{array}{c}0.7 \\
{[0.6-} \\
0.8]\end{array}$ & $\begin{array}{c}-4.0 \\
{[-10.1-} \\
2.1]\end{array}$ & $\begin{array}{c}0.7 \\
{[0.61-} \\
0.77]\end{array}$ & $\begin{array}{c}-2.0 \\
{[-7.8-} \\
3.8]\end{array}$ & $\begin{array}{c}0.8 \\
{[0.68-} \\
0.90]\end{array}$ & $\begin{array}{l}-7.7 * \\
{[-12.1} \\
--3.3]\end{array}$ & $\begin{array}{c}0.8 \\
{[0.7-} \\
0.9]\end{array}$ & $\begin{array}{c}-0.7 \\
{[-8.5-} \\
7.1]\end{array}$ & $\begin{array}{c}0.7 \\
{[0.6-} \\
0.8]\end{array}$ & $\begin{array}{l}-8.7 * \\
{[-13.6} \\
--3.8]\end{array}$ & $\begin{array}{c}0.7 \\
{[0.5-} \\
0.8]\end{array}$ & $\begin{array}{l}-11.6 \\
* \\
{[-20.6} \\
--2.7]\end{array}$ & $\begin{array}{c}0.7 \\
{[0.5-} \\
0.8]\end{array}$ & $\begin{array}{c}-11.6 \\
* \\
{[-22.1} \\
--1.1]\end{array}$ \\
\hline $\begin{array}{l}\text { CO } \\
\text { (ml/min) } \\
\text { A hep } \\
\text { Resist } \\
\text { (mmHg/ } \\
\mathrm{ml} \cdot \mathrm{min})\end{array}$ & $\begin{array}{c}116.7 \\
{[98.4-} \\
135.0] \\
20.8 \\
{[15.9-} \\
25.8]\end{array}$ & $\begin{array}{c}4.4 \\
{[-2.2-} \\
10.9] \\
0.8 \\
{[-13.9-} \\
15.5]\end{array}$ & $\begin{array}{c}120.3 \\
{[108.6-} \\
131.9] \\
20.3 \\
{[14.8-} \\
25.8]\end{array}$ & $\begin{array}{c}-0.8 \\
{[-4.5-} \\
2.9] \\
-17.4 \\
* \\
{[-32.8} \\
--2.1]\end{array}$ & $\begin{array}{c}106.7 \\
{[90.0-} \\
123.4] \\
25.1 \\
{[19.3-} \\
30.9]\end{array}$ & $\begin{array}{c}3.7 \\
{[-0.1-} \\
7.5] \\
-32.2 \\
* \\
{[-47.8} \\
-- \\
16.7]\end{array}$ & $\begin{array}{c}104.1 \\
{[85.33-} \\
122.9] \\
23.3 \\
{[16.1-} \\
30.5]\end{array}$ & $\begin{array}{c}2.3 \\
{[7.0-} \\
11.6] \\
-1.8 \\
{[-6.9-} \\
3.2]\end{array}$ & $\begin{array}{c}I 13.0 \\
{[100.0-} \\
126.0] \\
23.4 \\
{[18.6-} \\
28.2]\end{array}$ & $\begin{array}{c}1.3 \\
{[-3.8-} \\
6.4] \\
-21.8 \\
* \\
{[-35.2} \\
--8.4]\end{array}$ & $\begin{array}{c}119.7 \\
{[99.1-} \\
140.4] \\
20.7 \\
{[16.5-} \\
24.9]\end{array}$ & $\begin{array}{c}3.5 \\
{[-2.0-} \\
9.1] \\
-30.1 \\
* \\
{[-41.2} \\
-- \\
18.9]\end{array}$ & $\begin{array}{c}127.5 \\
{[103.8-} \\
151.2] \\
16.9 \\
{[13.5-} \\
20.2]\end{array}$ & $\begin{array}{c}0.4 \\
{[-6.0-} \\
6.9] \\
-18.8 \\
* \\
{[-28.1} \\
--9.5]\end{array}$ \\
\hline $\begin{array}{l}\text { A hep Flow } \\
(\mathrm{ml} / \mathrm{min})\end{array}$ & $\begin{array}{l}4.1 \\
{[3.2-} \\
5.1]\end{array}$ & $\begin{array}{c}0.9 \\
{[-12.0-} \\
13.9]\end{array}$ & $\begin{array}{c}4.4 \\
{[3.1-} \\
5.6]\end{array}$ & $\begin{array}{l}21.3 \\
{[-0.0-} \\
42.6]\end{array}$ & $\begin{array}{r}3.5 \\
{[2.6-} \\
4.4]\end{array}$ & $\begin{array}{c}47.5 \\
* \\
{[17.8-} \\
77.2]\end{array}$ & $\begin{array}{c}3.7 \\
{[2.7-} \\
4.7]\end{array}$ & $\begin{array}{c}3.3 \\
{[-2.6-} \\
9.1]\end{array}$ & $\begin{array}{c}3.5 \\
{[2.8-} \\
4.3]\end{array}$ & $\begin{array}{c}20.2 \\
* \\
{[3.2-} \\
37.2]\end{array}$ & $\begin{array}{c}3.9 \\
{[3.2-} \\
4.6]\end{array}$ & $\begin{array}{c}33.5 \\
* \\
{[11.6-} \\
55.5]\end{array}$ & $\begin{array}{c}5.1 \\
{[4.2-} \\
6.1]\end{array}$ & $\begin{array}{c}9.6 \\
{[-5.4-} \\
24.7]\end{array}$ \\
\hline $\begin{array}{l}\text { Port Resist } \\
(\mathrm{mmHg} / \\
\mathrm{ml} \cdot \mathrm{min})\end{array}$ & $\begin{array}{c}0.2 \\
{[0.1-} \\
0.2]\end{array}$ & $\begin{array}{c}2.8 \\
{[-1.9-} \\
7.5]\end{array}$ & $\begin{array}{c}0.1 \\
{[0.11-} \\
0.14]\end{array}$ & $\begin{array}{l}-20.3 \\
{[-34.5} \\
--6.2]\end{array}$ & $\begin{array}{c}0.1 \\
{[0.1-} \\
0.2]\end{array}$ & $\begin{array}{c}-33.8 \\
* \\
{[-46.8} \\
-- \\
20.8]\end{array}$ & $\begin{array}{c}0.2 \\
{[0.1-} \\
0.2]\end{array}$ & $\begin{array}{c}0.8 \\
{[-7.0-} \\
8.5]\end{array}$ & $\begin{array}{c}0.1 \\
{[0.1-} \\
0.2]\end{array}$ & $\begin{array}{c}-22.0 \\
* \\
{[-34.0} \\
-- \\
10.0]\end{array}$ & $\begin{array}{c}0.2 \\
{[0.1-} \\
0.2]\end{array}$ & $\begin{array}{c}-31.0 \\
* \\
{[-40.6} \\
-- \\
21.4]\end{array}$ & $\begin{array}{c}0.2 \\
{[0.1-} \\
0.2]\end{array}$ & $\begin{array}{c}-38.6 \\
* \\
{[-61.1} \\
-- \\
\mid 6.0]\end{array}$ \\
\hline $\begin{array}{l}\text { Port Flow } \\
(\mathrm{ml} / \mathrm{min})\end{array}$ & $\begin{array}{c}23.3 \\
{[20.1-} \\
26.5]\end{array}$ & $\begin{array}{c}-1.3 \\
{[-0.5-} \\
2.9]\end{array}$ & $\begin{array}{l}25.0 \\
{[21.7-} \\
28.3]\end{array}$ & $\begin{array}{c}\text { I8.3 } \\
* \\
{[2.0-} \\
34.5]\end{array}$ & $\begin{array}{c}25.3 \\
{[22.9-} \\
27.7]\end{array}$ & $\begin{array}{c}24.1 \\
* \\
{[13.3-} \\
34.9]\end{array}$ & $\begin{array}{c}21.7 \\
{[17.6-} \\
25.7]\end{array}$ & $\begin{array}{c}3.3 \\
{[-2.4-} \\
8.9]\end{array}$ & $\begin{array}{c}22.7 \\
{[20-6-} \\
24-8]\end{array}$ & $\begin{array}{c}23.9 \\
* \\
{[12.5-} \\
35.4]\end{array}$ & $\begin{array}{l}24.1 \\
{[21.1-} \\
27.1]\end{array}$ & $\begin{array}{c}29.2 \\
* \\
{[22.5-} \\
35.9]\end{array}$ & $\begin{array}{l}24.0 \\
{[21.1-} \\
26.9]\end{array}$ & $\begin{array}{l}15.9 \\
{[-4.6-} \\
36.5]\end{array}$ \\
\hline $\begin{array}{l}\text { Parench } \\
\text { Flow }\end{array}$ & $\begin{array}{r}135.2 \\
{[121.0-} \\
149.4]\end{array}$ & $\begin{array}{c}-3.9 \\
{[-14.0-} \\
6.2]\end{array}$ & $\begin{array}{c}151.8 \\
{[130.5-} \\
173.0]\end{array}$ & $\begin{array}{l}12.4 \\
* \\
{[2.5-} \\
22.2]\end{array}$ & $\begin{array}{l}133.7 \\
{[115.4-} \\
151.9]\end{array}$ & $\begin{array}{l}15.3 \\
* \\
{[9.8-} \\
20.8]\end{array}$ & $\begin{array}{l}131.3 \\
{[122.2-} \\
140.5]\end{array}$ & $\begin{array}{l}6.3 * \\
{[-1.9-} \\
14.5]\end{array}$ & $\begin{array}{c}126.3 \\
{[103.2} \\
- \\
.149 .4]\end{array}$ & $\begin{array}{c}28.8 \\
* \\
{[10.4-} \\
47.3]\end{array}$ & $\begin{array}{c}146.2 \\
{[118.2-} \\
174.1]\end{array}$ & $\begin{array}{l}15.0 \\
* \\
{[1.8-} \\
28.2]\end{array}$ & $\begin{array}{l}130.1 \\
{[114.1-} \\
146.0]\end{array}$ & $\begin{array}{c}6.8 \\
{[-10.0-} \\
23.6]\end{array}$ \\
\hline $\begin{array}{l}\text { Port } \\
\text { Pressure }\end{array}$ & $\begin{array}{c}6.3 \\
{[5.7-} \\
6.9]\end{array}$ & $\begin{array}{c}-0.8 \\
{[-5.7-} \\
4.1]\end{array}$ & $\begin{array}{c}6.1 \\
{[5.5-} \\
6.7]\end{array}$ & $\begin{array}{c}-1.3 \\
{[-6.9-} \\
4.3]\end{array}$ & $\begin{array}{c}6.1 \\
{[5.9-} \\
6.3]\end{array}$ & $\begin{array}{c}-6.2 \\
{[-13.6-} \\
1.3]\end{array}$ & $\begin{array}{c}5.7 \\
{[4.8-} \\
6.6]\end{array}$ & $\begin{array}{c}1.0 \\
{[-1-8-} \\
3-9]\end{array}$ & $\begin{array}{c}6.3 \\
{[5.8-} \\
6.7]\end{array}$ & $\begin{array}{c}-6.8 \\
{[-15.0-} \\
1.3]\end{array}$ & $\begin{array}{l}6.4 \\
{[5.7-} \\
7.1]\end{array}$ & $\begin{array}{c}-7.5 \\
{[-14.2} \\
--0.1]\end{array}$ & $\begin{array}{c}6.3 \\
{[5.3-} \\
7.4]\end{array}$ & $\begin{array}{c}-11.2 \\
{[-24.8-} \\
2.4]\end{array}$ \\
\hline $\begin{array}{l}\text { Fract Liver } \\
\text { Flow } \\
\text { (\%) }\end{array}$ & $\begin{array}{c}23.8 \\
{[20.6-} \\
27.0]\end{array}$ & $\begin{array}{c}-4.8 \\
{[-12.6-} \\
3.0]\end{array}$ & $\begin{array}{l}24.5 \\
{[21.7-} \\
27.3]\end{array}$ & $\begin{array}{c}19.3 \\
* \\
{[5.6-} \\
32.9]\end{array}$ & $\begin{array}{l}27.5 \\
{[23.7-} \\
31.3]\end{array}$ & $\begin{array}{c}22.9 \\
* \\
{[11.6-} \\
34.2]\end{array}$ & $\begin{array}{r}24.5 \\
{[19.5-} \\
29.6]\end{array}$ & $\begin{array}{c}1.4 \\
{[-8.6-} \\
11.4]\end{array}$ & $\begin{array}{l}23.6 \\
{[19.2-} \\
28.0]\end{array}$ & $\begin{array}{c}22.0 \\
* \\
{[10.9-} \\
33.2]\end{array}$ & $\begin{array}{l}23.7 \\
{[21.3-} \\
26.0]\end{array}$ & $\begin{array}{c}25.5 \\
* \\
{[15.9-} \\
35.1]\end{array}$ & $\begin{array}{c}23.3 \\
{[18.1-} \\
28.5]\end{array}$ & $\begin{array}{r}14.5 \\
{[-3.7-} \\
32.7]\end{array}$ \\
\hline
\end{tabular}

Significant changes ( $p<0.05$; Wilcoxon rank sum test) are marked with $*$ MAP = mean arterial pressure $(\mathrm{mm} \mathrm{Hg}), \mathrm{HR}=$ heart rate $(\mathrm{I} / \mathrm{min}), \mathrm{SVR}=$

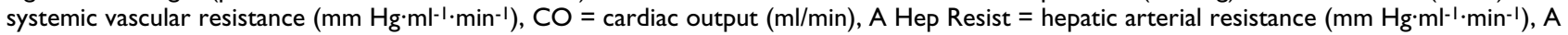
Hep Flow $=$ hepatic arterial flow $(\mathrm{ml} / \mathrm{min})$, Port Resist $=$ portal venous resistance $\left(\mathrm{mm} \mathrm{Hg} \cdot \mathrm{ml}^{-1} \cdot \mathrm{min}^{-1}\right)$, Port Flow $=$ portal venous flow $(\mathrm{ml} / \mathrm{min})$, Parench Flow $=$ parenchymal flow $(\mathrm{ml} / \mathrm{min})$, Port Pressure $=$ portal venous pressure $(\mathrm{mm} \mathrm{Hg})$. Fract Liver Flow $=$ fractional liver flow $($ arterial liver flow + portal liver flow/cardiac output) $(\%)$.

a decrease (Figure 1). From the data we calculated the fractional liver flow (portal venous + hepatic arterial blood flow $/$ cardiac output $=$ proportion of cardiac output passing through the liver, Figure 3, panel 3). In sil10 it increased at 10,30 , and 60 min by $16 \%, 30 \%$, and $23 \%$. In var10 it increased by $14 \%, 24 \%$, and $26 \%$.

\section{Discussion}

This is the first experimental study in which the effect of different low doses of two inhibitors of phosphodieste- rase 5 on hepatic and systemic hemodynamics was investigated up to $60 \mathrm{~min}$ after intravenous injection. We showed a distinct effect of the PDE-5 inhibitors, sildenafil and vardenafil, at low doses on the hemodynamics of the liver: 1. Portal venous, hepatic arterial, and hepatic parenchymal blood flow increased. 2. Portal venous and hepatic arterial resistance decreased. 3. Portal pressure showed a trend towards decreasing. 4. Sildenafil decreases mean arterial pressure by $<4 \%$ and vardenafil by $\leq 11 \%$. 5 . Heart rate, central venous pressure and cardiac output 
remained constant. 6. Fractional liver blood flow increased. Combining the data from Figure 1 and Table 1 we may conclude, that the $10 \mu \mathrm{g} / \mathrm{kg}$ dose is optimal for a more or less selective effect on hepatic hemodynamics, because it only slightly affects systemic hemodynamics parameters. These results contribute to the understanding of the physiology of liver hemodynamics and have potential implications for the treatment of portal hypertension.

About 25\% of the cardiac output passes through the liver. Liver blood supply is provided by the hepatic artery (25 $30 \%$ ) and the portal vein (up to 75\%) [3-5]. Blood flow rates in both vessels closely correlate and are regulated by multiple factors. A decline in portal venous blood flow enhances hepatic arterial flow by reducing hepatic arterial vascular resistance and vice versa. This mechanism known as hepatic arterial buffer response maintains the total liver blood supply [45].

Our data show that PDE-5 inhibitors - after intravenous application - increase both arterial and portal liver blood flow. The local vasodilation induced by these drugs abolishes - at least in this experimental setting - the hepatic buffer response. However, this effect is more than compensated by the decrease of portal resistance. This is reflected by the fact that the parenchymal liver flow is increased by $>15 \%$ and that the portal pressure does not increase but rather shows a tendency towards decrease (almost significant in the var10 group). Even if there is a dilation of splanchnic vessels leading to an increased blood flow towards the liver, all effects of PDE-5 inhibitors on extrahepatic arterial vessels are more than compensated by their intrahepatic effects. There are conflicting data whether or not an increase of portal flow alone increases portal pressure. Lee et al. [46] clearly demonstrated that a postprandial increase of portal flow leads to an increase of portal pressure even in normal liver. This increase was much more pronounced in cirrhotics. However, even in cirrhotic liver organic nitrates can modulate the postprandial increase of portal pressure [47]. Jiao et al. [48] observed a dramatic increase of portal pressure following increase of portal flow in the isolated perfused porcine liver. Recently, Zipprich et al. [49] investigated the effect of a selective increase of flow in the hepatic artery or the portal vein on portal pressure in normal and cirrhotic rat liver. Their system with in-situ perfused rat liver is in many respects comparable to our model. They found, that an increase of arterial perfusion increases portal pressure both in normal and in cirrhotic liver, whereas an increase of portal flow increases portal pressure only in cirrhotic liver. Despite an increase of both arterial and portal hepatic perfusion we observed no increase of portal pressure. Therefore, our data suggest that PDE-5 inhibitors act on intrahepatic structures beyond the point where portal and arterial flows merge. It may be speculated that even low levels of PDE-5 inhibitors increase the local cGMP concentration in the stellate cells and induce a dilation of the sinusoids.

There are only few papers which dealt with hemodynamic effects of PDE- 5 inhibitors in animals or humans with cirrhosis [50-52]. However, these authors focused on systemic or renal effects, the hepatic hemodynamics were not investigated. In addition, the authors used very high doses of the PDE-5 inhibitors.

Loureiro-Silva et al. investigated the effect of sildenafil on hepatic hemodynamics in normal and cirrhotic rat liver [23]. They found an increased expression of both PDE-5 and soluble guanylate cyclase in cirrhotic liver. For functional studies they used perfused liver, methoxamine was used for preconstriction of the intrahepatic circulation. Endogenous NO production was inhibited by the NO synthase inhibitor L-NMMA (N-monomethyl-L-arginine) and vasodilation was induced by the NO donor SNAP (SNitroso-N-Acetylpenicillamine). In this setting a reduced vasodilatory response to SNAP in cirrhosis could be demonstrated, which is corrected (or even overcompensated) by $10^{-8} \mathrm{M}$ sildenafil. While this approach yields information on the regulation of the sinusoidal tonus, it is difficult to translate these data to patients with portal hypertension.

Our data partially confirm the data obtained by Colle et al. [41] who studied the effect of sildenafil on mesenteric blood flow, mean arterial pressure, and portal venous pressure at doses ranging from $10-10,000 \mu \mathrm{g} / \mathrm{kg}$ injected into the mesenteric artery or intravenously. In contrast to our experimental protocol, the authors monitored the parameters for up to $10 \mathrm{~min}$ after the injection only and used repetitive doses after 10 min washout. In this experimental setting it was not possible to detect the changes in hemodynamic parameters that we describe. Nevertheless, they also found the transient dip in arterial blood pressure after injection of the drug. At the lowest doses of 10 and $100 \mu \mathrm{g} / \mathrm{kg}$ given intravenously the relative decrease of mean arterial pressure was about 3\%. The relative increase of mesenteric blood flow was $\leq 10 \%$, and no change of portal venous pressure was observed. The initial drop of MAP and SVR in our study occurred presumably due to a transiently higher drug concentration immediately after intravenous injection. From the results obtained with high doses ( 1000 and $10000 \mu \mathrm{g} / \mathrm{kg}$ given intraarterially or intravenously) used in the study by Colle and coworkers no conclusions can be drawn whether or not PDE-5 inhibitors may be a risk for variceal bleeding in liver cirrhosis.

The present study in rats with normal liver demonstrates the influence of low-dose PDE-5 inhibitors on hepatic blood flow. In this setting they marginally interfere with 
systemic hemodynamics. With regard to cardiac output we calculated the values for fractional hepatic blood flow after drug injection (Figure 3, panel 3). These results revealed an increase in selective liver blood supply compared to baseline by 20 - 30\%. To the best of our knowledge to date this has not been shown for any other drug. In our animal study the ideal dose for short term use is in the range of $10 \mu \mathrm{g} / \mathrm{kg}$. This is much less than the standard dose of vardenafil (10 mg) or sildenafil (50 mg) used for therapy of erectile dysfunction in the clinical setting. With a dose in the range of $10 \mu \mathrm{g} / \mathrm{kg}$ the systemic hemodynamic effects of PDE-5 inhibitors could be neglected.

Our data are well in line with those of Lee et al [39]. These authors described an increase of cGMP levels in liver veins of cirrhotics after oral administration of $50 \mathrm{mg}$ sildenafil but not in peripheral veins leading to a significant decrease of sinusoidal resistance but not of peripheral vascular resistance. Therefore it can be concluded that conditions may exist (oral vs. intravenous applications, low doses) in which hemodynamic effects of PDE 5 inhibitors prevail in the liver leaving systemic circulation more or less unaffected. Comparing oral administration to intravenous application, a suspended arterial buffer response was demonstrated in rats in this study. In healthy and cirrhotic human livers, we have shown that after oral administration the buffer response stays intact [37].

Our findings suggest a distinct effect of PDE-5 inhibitors on hepatic hemodynamics. However, we cannot definitively answer the question whether or not PDE-5 inhibitors can be used to treat portal hypertension (or other conditions such as ischemia/reperfusion injury). We are presently examining the responses to low doses of PDE-5 inhibitors in animals with portal hypertension. If PDE-5 inhibitors are beneficial in this model, patients with liver cirrhosis should be evaluated. The measures of efficacy should be the hepatovenous pressure gradient and clinical end points, such as bleeding or re-bleeding from esophageal varices.

\section{Conclusion}

PDE-5 inhibitors at low doses have distinct effects on liver hemodynamics. Portal flow and parenchymal flow increase without enhancement of portal pressure in normal rat liver. The compromised NO-bioavailability in the cirrhotic liver leads to a constriction of the sinusoids, which contributes to the functional component of portal hypertension. It may be anticipated that this effect can be reversed by application of PDE-5 inhibitors.

\section{Abbreviations}

PDE-5: phosphodiesterase-5; NO: nitric oxide; cGMP: cyclic guanosine monophosphate; sil: sildenafil; var: vardenafil.

\section{Competing interests}

The University of Freiburg holds a European patent: Use of inhibitors of phosphodiesterase 5 for therapy or prophylaxis of portal hypertension.

\section{Authors' contributions}

PD and WK had the idea to perform this study and conceived its details. LH and RS performed the experiments and contributed to the concept of the study. PD and WK wrote the manuscript, PD did the statistical evaluations. HEB participated in writing the manuscript and coordination of the study. TD provided us the drugs and participated in the concept of the study. BJP participated in the concept of the study. LH and PD contributed equally to the work. All authors read and approved the final manuscript.

\section{References}

I. Wiest R, Groszmann RJ: The paradox of nitric oxide in cirrhosis and portal hypertension: too much, not enough. Hepatology 2002, 35:478-49l.

2. Blei AT: Portal hypertension and its complications. Curr Opin Gastroenterol 2007, 23:275-282.

3. DeLeve LD: Hepatic microvasculature in liver injury. Semin Liver Dis 2007, 27:390-400.

4. Pannen BH: New insights into the regulation of hepatic blood flow after ischemia and reperfusion. Anesth Analg 2002, 94: $1448-1457$.

5. Iwakiri Y, Groszmann RJ: The hyperdynamic circulation of chronic liver diseases: from the patient to the molecule. Hepatology 2006, 43:SI2I-SI3I.

6. Shah V, Haddad FG, Garcia-Cardena G, Frangos JA, Mennone A, Groszmann RJ, Sessa WC: Liver Sinusoidal Endothelial Cells Are Responsible for Nitric Oxide Modulation of Resistance in the Hepatic Sinusoids. J Clin Invest 1997, 100:2923-2930.

7. Rodriguez-Vilarrupla A, Fernandez M, Bosch J, Garcia-Pagan JC: Current concepts on the pathophysiology of portal hypertension. Ann Hepatol 2007, 6:28-36.

8. Sessa WC: eNOS at a glance. J Cell Sci 2004, I I 7:2427-2429.

9. Bellamy TC, Wood J, Garthwaite J: On the activation of soluble guanylyl cyclase by nitric oxide. Proc Natl Acad Sci USA 2002, 99:507-510.

10. Matsumoto T, Kobayashi T, Kamata K: Phosphodiesterases in the vascular system. J Smooth Muscle Res 2003, 39:67-86.

II. Prisant LM: Phosphodiesterase-5 inhibitors and their hemodynamic effects. Curr Hypertens Rep 2006, 8:345-35I.

12. Pannen $B H$, Bauer M: Differential regulation of hepatic arterial and portal venous vascular resistance by nitric oxide and carbon monoxide in rats. Life Sci 1998, 62:2025-2033.

13. Bauer M, Zhang JX, Bauer I, Clemens MG: ET-I induced alterations of hepatic microcirculation: sinusoidal and extrasinusoidal sites of action. Am J Physiol Gastrointest Liver Physiol 1994, 267:GI43-GI49.

14. Angus PW: Role of endothelin in systemic and portal resistance in cirrhosis. Gut 2006, 55:1230-1232.

15. Schmidt R, Tritschler E, Hoetzel A, Loop T, Humar M, Halverscheid L, Geiger KK, Pannen BH: Heme oxygenase-I induction by the clinically used anesthetic isoflurane protects rat livers from ischemia/reperfusion injury. Ann Surg 2007, 245:93I-942.

16. Hoetzel A, Welle A, Schmidt R, Loop T, Humar M, Ryter SW, Geiger KK, Choi AM, Pannen BH: Nitric oxide-deficiency regulates hepatic heme oxygenase-I. Nitric Oxide 2008, 18:61-69.

17. Iwakiri $Y$, Groszmann RJ: Vascular endothelial dysfunction in cirrhosis. J Hepatol 2007, 46:927-934.

18. Bosch J: Vascular deterioration in cirrhosis: the big picture. J Clin Gastroenterol 2007, 41 :S247-S253. 
19. Zipprich A: Hemodynamics in the isolated cirrhotic liver. J Clin Gastroenterol 2007, 41:S254-S258.

20. Malyshev E, Tazi KA, Moreau R, Lebrec D: Discrepant effects of inducible nitric oxide synthase modulation on systemic and splanchnic endothelial nitric oxide synthase activity and expression in cirrhotic rats. J Gastroenterol Hepatol 2007, 22:2195-2201.

21. Sancho-Bru P, Bataller R, Gasull X, Colmenero J, Khurdayan V, Gual A, Nicolas JM, Arroyo V, Gines P: Genomic and functional characterization of stellate cells isolated from human cirrhotic livers. J Hepatol 2005, 43:272-282.

22. Davies NA, Hodges SJ, Pitsillides AA, Mookerjee RP, Jalan R, Mehdizadeh S: Hepatic guanylate cyclase activity is decreased in a model of cirrhosis: a quantitative cytochemistry study. FEBS Lett 2006, 580:2I23-2I28.

23. Loureiro-Silva MR, Iwakiri Y, Abraldes JG, Haq O, Groszmann RJ: Increased phosphodiesterase-5 expression is involved in the decreased vasodilator response to nitric oxide in cirrhotic rat livers. J Hepatol 2006, 44:886-893.

24. Loureiro-Silva MR, Cadelina GW, Groszmann RJ: Deficit in nitric oxide production in cirrhotic rat livers is located in the sinusoidal and postsinusoidal areas. Am J Physiol Gastrointest Liver Physiol 2003, 284:G567-G574.

25. Kawada N, Tran-Thi TA, Klein H, Decker K: The contraction of hepatic stellate (Ito) cells stimulated with vasoactive substances. Possible involvement of endothelin I and nitric oxide in the regulation of the sinusoidal tonus. Eur J Biochem 1993, 213:815-823.

26. Tsai MH, Iwakiri Y, Cadelina G, Sessa WC, Groszmann RJ: Mesenteric vasoconstriction triggers nitric oxide overproduction in the superior mesenteric artery of portal hypertensive rats. Gastroenterology 2003, 125:|452-146I.

27. Wiest R, Shah V, Sessa WC, Groszmann RJ: NO overproduction by eNOS precedes hyperdynamic splanchnic circulation in portal hypertensive rats. Am J Physiol 1999, 276:G I043-GI05I.

28. Carini R, Albano E: Recent insights on the mechanisms of liver preconditioning. Gastroenterology 2003, I 25:|480-|49|.

29. Peralta C, Hotter G, Closa D, Gelpi E, Bulbena O, Rosello-Catafau J: Protective effect of preconditioning on the injury associated to hepatic ischemia-reperfusion in the rat: role of nitric oxide and adenosine. Hepatology 1997, 25:934-937.

30. Fiorucci S, Antonelli E, Brancaleone V, Sanpaolo L, Orlandi S, Distrutti E, Acuto G, Clerici C, Baldoni M, Del Soldato P, et al.: NCX-a nitric oxide-releasing derivative of ursodeoxycholic acid, ameliorates portal hypertension and lowers norepinephrineinduced intrahepatic resistance in the isolated and perfused rat liver. J Hepatol 1000, 39:932-939.

31. Yu Q, Shao R, Qian HS, George SE, Rockey DC: Gene transfer of the neuronal NO synthase isoform to cirrhotic rat liver ameliorates portal hypertension. I Clin Invest 2000, 105:74I-748.

32. Casteele M Van de, Omasta A, Janssens S, Roskams T, Desmet V, Nevens $F$, Fevery J: In vivo gene transfer of endothelial nitric oxide synthase decreases portal pressure in anaesthetised carbon tetrachloride cirrhotic rats. Gut 2002, 5 I:440-445.

33. Morales-Ruiz M, Cejudo-Martin P, Fernandez-Varo G, Tugues S, Ros J, Angeli P, Rivera F, Arroyo V, Rodes J, Sessa WC, et al.: Transduction of the liver with activated Akt normalizes portal pressure in cirrhotic rats. Gastroenterology 2003, 125:522-53I.

34. Trebicka J, Hennenberg M, Laleman W, Shelest N, Biecker E, Schepke M, Nevens F, Sauerbruch T, Heller J: Atorvastatin lowers portal pressure in cirrhotic rats by inhibition of RhoA/Rho-kinase and activation of endothelial nitric oxide synthase. Hepatology 2007, 46:242-253

35. Zafra C, Abraldes JG, Turnes J, Berzigotti A, Fernandez M, GarcaPagan JC, Rodes J, Bosch J: Simvastatin enhances hepatic nitric oxide production and decreases the hepatic vascular tone in patients with cirrhosis. Gastroenterology 2004, I 26:749-755.

36. Laleman W, Van Landeghem L, Van dEI, Zeegers M, Fevery J, Nevens F: Nitroflurbiprofen, a nitric oxide-releasing cyclooxygenase inhibitor, improves cirrhotic portal hypertension in rats. Gastroenterology 2007, I 32:709-7| 9 .

37. Deibert P, Schumacher YO, Ruecker G, Opitz OG, Blum HE, Rössle $M$, Kreisel W: Effect of vardenafil, an inhibitor of phosphodiesterase-5, on portal haemodynamics in normal and cir- rhotic liver -- results of a pilot study. Aliment Pharmacol Ther 2006, 23: $12 \mid-128$.

38. Deibert P, Bremer H, Roessle M, Kurz-Schmieg AK, Kreisel W: PDE5 inhibitors lower portal and pulmonary pressure in portopulmonary hypertension. Eur Respir J 2007, 29:220-22I.

39. Lee KC, Yang YY, Wang YW, Hou MC, Lee FY, Lin HC, Lee SD: Acute administration of sildenafil enhances hepatic cyclic guanosine monophosphate production and reduces hepatic sinusoid resistance in cirrhotic patients. Hepatol Res 2008, 38: II86-II 93.

40. Clemmesen JO, Giraldi A, Ott P, Dalhoff K, Hansen BA, Larsen FS: Sildenafil does not influence hepatic venous pressure gradient in patients with cirrhosis. World J Gastroenterol 2008, 14:6208-62 I 2 .

4I. Colle I, De Vriese AS, Van Vlierberghe H, Lameire NH, DeVos M: Systemic and splanchnic haemodynamic effects of sildenafil in an in vivo animal model of cirrhosis support for a risk in cirrhotic patients. Liver Int 2004, 24:63-68.

42. Wang YW, Lin HC, Yang YY, Hou MC, Lee SD: Sildenafil decreased pulmonary arterial pressure but may have exacerbated portal hypertension in a patient with cirrhosis and portopulmonary hypertension. J Gastroenterol 2006, 4I:593-597.

43. Tzathas C, Christidou A, Ladas SD: Sildenafil (viagra) is a risk factor for acute variceal bleeding. Am J Gastroenterol 2002, 97: 1856.

44. Finley DS, Lugo B, Ridgway J, Teng W, Imagawa DK: Fatal variceal rupture after sildenafil use: report of a case. Curr Surg 2005, 62:55-56.

45. Kollmar O, Corsten M, Scheuer C, Vollmar B, Schilling MK, Menger MD: Portal branch ligation induces a hepatic arterial buffer response, microvascular remodeling, normoxygenation, and cell proliferation in portal blood-deprived liver tissue. Am J Physiol Gastrointest Liver Physiol 2007, 292:G I 534-GI 542.

46. Lee SS, Hadengue A, Moreau R, Sayegh R, Hillon P, Lebrec D: Postprandial hemodynamic responses in patients with cirrhosis. Hepatology 1988 May -Jun 1908:647-65I.

47. Bellis L, Berzigotti A, Abraldes JG, Moitinho E, Garcia-Pagan JC, Bosch J, Rodes J: Low doses of isosorbide mononitrate attenuate the postprandial increase in portal pressure in patients with cirrhosis. Hepatology 2003, 37:378-384.

48. Jiao LR, Inglott FS, Mathie RT, Habib NA: The effect of augmenting portal venous inflow on intrahepatic pressure and resistance in the isolated perfused porcine liver. Hepatogastroenterology 200I, 48: I0I I-I0I4.

49. Zipprich A, Loureiro-Silva MR, D'Silva I, Groszmann RJ: The role of hepatic arterial flow on portal venous and hepatic venous wedged pressure in the isolated perfused $\mathrm{CCl}$ 4-cirrhotic liver. Am J Physiol Gastrointest Liver Physiol 2008, 295:G1 97-G202.

50. Ghali-Goul R, Tahseldar-Roumieh R, Sabra M: Effect of chronic administration of sildenafil on sodium retention and on the hemodynamic complications associated with liver cirrhosis in the rat. Eur J Pharmacol 2007, 572:49-56.

5I. Tahseldar-Roumieh R, Ghali-Goul R, Lugnier C, Sabra M: Effect of phosphodiesterase 5 inhibitor on alteration in smooth muscle sensitivity and renal function in rats with cirrhosis. Am J Physiol Heart Circ Physiol 2006, 290:H48I-H4889.

52. Thiesson HC, Jensen BL, Jespersen B, Schaffalitzky de Muckadell OB Bistrup C, Walter S, Ottosen PD, Veje A, Skøtt O: Inhibition of cGMP-specific phosphodiesterase type 5 reduces sodium excretion and arterial blood pressure in patients with $\mathrm{NaCl}$ retention and ascites. Am J Physiol Renal Physiol 2005, 288:FI044-FI052.

\section{Pre-publication history}

The pre-publication history for this paper can be accessed here:

http://www.biomedcentral.com/1471-230X/9/69/pre pub 